\title{
Corela
}

Cognition, représentation, langage

8-2 $\mid 2010$

Vol. $8, \mathrm{n}^{\circ} 2$

\section{Étudier des structures de discours : préoccupations pratiques et méthodologiques}

Marie-Paule Jacques et Thierry Poibeau

\section{OpenEdition}

\section{Journals}

Édition électronique

URL : http://journals.openedition.org/corela/1855

DOI : $10.4000 /$ corela. 1855

ISSN : $1638-573 \mathrm{X}$

\section{Éditeur}

Cercle linguistique du Centre et de I'Ouest - CerLICO

\section{Référence électronique}

Marie-Paule Jacques et Thierry Poibeau, «Étudier des structures de discours : préoccupations pratiques et méthodologiques », Corela [En ligne], 8-2 | 2010, mis en ligne le 27 octobre 2010, consulté le 20 avril 2019. URL : http://journals.openedition.org/corela/1855 ; DOI : 10.4000/corela.1855

Ce document a été généré automatiquement le 20 avril 2019

\section{(c) (i) (2)(2)}

Corela - cognition, représentation, langage est mis à disposition selon les termes de la licence Creative Commons Attribution - Pas d'Utilisation Commerciale - Partage dans les Mêmes Conditions 4.0 International. 


\section{Étudier des structures de discours : préoccupations pratiques et méthodologiques}

Marie-Paule Jacques et Thierry Poibeau

\section{Introduction}

1 L'analyse linguistique, quand elle ne porte pas sur l'étude d'une forme particulière mais sur un phénomène sémantique, discursif ou pragmatique est d'emblée confrontée à un problème : comment cerner l'objet d'étude en lui-même?

2 En effet, lorsque l'objet d'analyse est une forme, le repérage des occurrences ne pose guère de problème: il s'agit simplement de rechercher cette forme et ses éventuelles flexions dans les textes. Les choses se compliquent dès lors que l'analyse cible un niveau sémantico-pragmatique, qui requiert une interprétation, c'est-à-dire l'attribution d'un sens ou d'un effet pragmatique aux formes du texte ${ }^{1}$. Elles se compliquent encore davantage si l'on aborde un phénomène ou un objet linguistique non pas selon une approche sémasiologique mais selon une approche onomasiologique, c'est-à-dire si l'on prend comme point de départ un certain sens ou un certain effet pragmatique et comme objectif la mise en évidence des formes linguistiques susceptibles de produire ce sens ou cet effet pragmatique. La difficulté essentielle réside alors dans le recueil des séquences textuelles qui manifestent sans conteste le phénomène ou objet à l'étude, puisque ce recueil ne peut s'appuyer sur autre chose que l'interprétation.

\subsection{Définir son objet d'étude}

Dans cet article, nous prendrons prétexte de l'analyse en corpus des modalités d'expression des procédures pour mener une réflexion d'ordre méthodologique. L'étude de l'expression des procédures, et plus généralement, l'analyse en corpus de phénomènes linguistiques qui ressortissent au niveau du discours (par opposition au niveau du lexique 
ou de la phrase) fait émerger diverses questions de méthode, au premier rang desquelles la simple identification du ou des phénomènes étudiés dans les textes.

4 S'agissant des procédures, c'est une difficulté qui a été bien soulignée par M.-P. PéryWoodley :

« Qu'appelle-t-on discours procédural, texte injonctif, texte à consignes ? Plutôt que de tenter une définition in abstracto, je poserai ici la question en référence à un projet particulier : la constitution d'un corpus de textes à partir desquels examiner les réalisations spécifiques des consignes. Impossible de prendre pour critère de sélection des traits linguistiques particuliers, présence d'impératifs, par exemple: cela reviendrait à répondre a priori à la question posée et exclurait toute possibilité de découverte de formulations inattendues. Il est clair que pour que l'examen des réalisations linguistiques soit réellement ouvert, les textes doivent être sélectionnés sur des bases autres que des critères linguistiques. » (Péry-Woodley, $2001: 31)$.

Comment ne pas s'enfermer dans cette double contrainte: d'un côté, on ne peut constituer un corpus pour l'analyse d'un certain phénomène linguistique sans avoir déjà une idée de la façon dont ce phénomène sera réalisé dans les textes; d'un autre côté, la détermination des caractéristiques formelles de l'objet est un objectif de l'étude et ne doit donc pas être arrêtée au commencement même de l'étude. Nombre de linguistes «de corpus » menant une recherche sur des objets discursifs se reconnaîtront dans une telle interrogation qui touche les fondements mêmes des analyses linguistiques. L'enjeu est effectivement de taille car il concerne la validité d'une linguistique de corpus pour les objets linguistiques non formels, ce que sont en définitive la plupart - sinon tous - les phénomènes de discours que l'on range sous l'étiquette pragmatique.

Il paraît dès lors nécessaire d'asseoir l'étude de tels objets sur une méthodologie rigoureuse dont les étapes et les choix soient clairement motivés par le souci de ne pas biaiser les résultats dès la phase de constitution des données. Nous considérons que celleci n'est pas un préalable de l'étude linguistique, mais qu'elle en est partie prenante. Nous voulons montrer ici, à travers le cas particulier des séquences procédurales, comment la constitution des données, loin d'être anecdotique, peut et doit être intégrée à l'étude même, en tant qu'un des moments de l'analyse qui permet in fine de produire une description de l'objet.

\subsection{Questions de méthode}

Il s'agit ici de partir d'un cas concret pour essayer de mettre en évidence des éléments de méthode qui sont, nous l'espérons, réutilisables par d'autres études de ce type.

8 En entamant le travail sur les procédures, nous avions en tête la problématique posée par M.-P. Péry-Woodley et nous avons cherché à y répondre : comment définir notre objet d'étude, comment identifier le phénomène dans les textes, donc, en premier lieu, quels textes choisir?

9 Pour le choix des textes pertinents, c'est-à-dire susceptibles de contenir le phénomène visé, on propose de s'appuyer en priorité sur des textes dont la nature serait validée par une autorité neutre et extérieure au projet, même si cette solution n'est pas toujours possible. Précisons d'emblée que l'inventaire des genres susceptibles d'inclure l'expression de procédures n'est pas la principale question de cet article et que nous n'apporterons aucune conclusion en termes de constitution de corpus - sinon la 
préconisation du recours à un jugement extérieur pour l'adéquation des textes à l'objectif de recherche ${ }^{2}$. Nous nous focalisons sur le recueil des occurrences dans les textes.

C'est alors la finesse de l'analyse qui peut poser problème. Selon la tâche considérée (et particulièrement ici l'analyse de la notion de procédure), l'empan de texte pertinent varie. S'agit-il du document, du paragraphe, de la phrase?

11 On prône donc ici une analyse initiale impliquant plusieurs analystes (linguistes, experts du domaine, utilisateurs), sans guide prédéfini. Le but de cette étape est d'enrichir les documents avec une annotation de type "surlignage » du phénomène étudié. Une phase de confrontation des analyses ainsi produites permet ensuite de repérer les cas d'accord (dans le cas présent, accord sur ce qui est procédural et sur ce qui est non procédural) et les cas de désaccord. Il s'agit alors de déterminer les principales raisons de ces désaccords. En fait, chaque analyste se donne implicitement des règles qui peuvent différer d'une personne à l'autre : ce sont essentiellement ces cas de désaccords qui sont intéressants ${ }^{3}$. Le fait de mettre au jour ces cas de désaccords a un double but :

- D'une part, identifier de manière explicite les zones problématiques, les caractériser afin d'aboutir à un guide d'annotation faisant consensus et permettant d'obtenir une annotation stable d'un annotateur à l'autre.

- D'autre part, analyser linguistiquement les éléments de désaccord afin de mettre en évidence les raisons de choix différents. On espère pouvoir ainsi profiter de l'annotation libre pour cerner les "cas limites", où une séquence textuelle peut être interprétée différemment suivant le point de vue adopté.

12 Ce dernier point nous semble particulièrement important. L'analyse de discours porte rarement sur des objets « tout noirs ou tout blancs » : on est au contraire dans le domaine du clair-obscur, chaque objet linguistique dégage un ensemble de potentiels qui entre en interaction avec le contexte et peut être interprété avec des nuances différentes suivant le lecteur. Il ne s'agit pas de défendre ici un arbitraire complet mais il est important de prendre en compte cette zone intermédiaire. Elle ne doit pas pour autant faire oublier le fait que la plupart des cas sont annotés sans hésitation, et qu'on aboutit pour ceux-ci à un accord parfait, quel que soit le nombre d'annotateurs.

Dans cet article, nous souhaitons illustrer les points de méthodes mis ici en avant par l'étude des textes procéduraux. On verra qu'à côté de zones textuelles clairement procédurales (ou à l'inverse, non procédurales, comme des séquences clairement descriptives par exemple) subsistent d'autres plus difficiles à caractériser, comme des normes ou des règlements.

\subsection{Plan de l'article}

14 La section 2 présente un rapide panorama de la question de l'annotation du niveau discursif tandis que la section 3 donne un aperçu des recherches visant à analyser la notion de procéduralité, que ce soit dans une perspective (psycho-)linguistique ou automatique. Dans la section 4, nous décrivons les principales caractéristiques de notre stratégie globale de recueil et d'analyse des données. La section 5 expose de façon concrète comment cette stratégie largement appuyée sur un travail d'annotation manuelle a été mise en œuvre dans le cas des textes procéduraux. Nous examinons certains cas d'accord et de désaccord entre annotateurs, et ce que cet examen nous apprend quant au phénomène étudié. Enfin, la conclusion nous permet d'élargir notre vision à d'autres objets discursivo-pragmatiques. 


\section{2. État de l'art}

Plusieurs études ont souligné la difficulté à définir l'objet d'étude quand on s'intéresse au niveau discursif. La citation de M.-P Péry-Woodley (2001) en introduction résume bien cette problématique: constituer puis annoter un corpus n'est pas une tâche triviale quand on travaille sur le niveau sémantique ou discursif. Nous reprenons ici quelques travaux sur la question en nous situant dans une perspective de linguistique de corpus (c'est-à-dire dans la perspective d'études à partir de données textuelles attestées et représentatives de la diversité des usages). Les questions abordées ne visent pas tant la constitution du corpus lui-même que les étapes préliminaires : quels types de données considérer, comment vérifier que ces données sont pertinentes et qu'elles sont représentatives de la diversité des usages.

M.-P. Péry-Woodley s'interroge dans plusieurs articles sur la définition de l'objet d'étude quand le chercheur s'intéresse au niveau discursif. Cette problématique est vive aussi dans le monde anglo-saxon (voir Péry-Woodley et Scott, 2006 pour un aperçu général), qui possède une longue tradition à la fois en matière de linguistique de corpus, d'études de discours et de stylistique (Stede et al. 2007 présentent ainsi un ensemble de corpus annotés au niveau discursif). Péry-Woodley $(2005$, p. 202) rappelle le propos de Leech et al. (1997), pour qui l'annotation stylistique de textes est fragile (the "last secure type of annotation") du fait de l'absence de critères clairs permettant de caractériser a priori les annotations pertinentes à porter sur le texte ("the absence of clear and concrete criteria to identify categories inevitably leads to a considerable degree of indeterminacy"). Si l'analyse stylistique ne pose pas exactement les mêmes questions que l'analyse de structures discursives, les problèmes sont largement partagés.

M.P. Péry-Woodley (2005) poursuit son investigation en prônant l'utilisation d'outils informatiques permettant l'annotation de larges corpus, sans présélection des formes, afin de s'appuyer autant que faire se peut sur des modes opératoires en largeur, objectifs et reproductibles. Cependant, le fait que les objets discursifs en question sont difficiles à repérer automatiquement et, tout simplement, à définir, laisse le problème largement ouvert.

18 De fait, jusqu'à présent, les études qui mêlent linguistique de corpus et niveau discursif ont plutôt porté sur des catégories relativement facilement repérables. Nous laissons de côté le cas de l'anaphore qui constitue un domaine de recherche en soi mais nous pouvons citer le travail de S. Leroy (2004) sur l'antonomase ou de Rinck et Tutin (2007) sur l'analyse de séquences entre guillemets. À partir d'un repérage de séquences ou formes linguistiques précises (les noms de personnes dans un cas, les passages entre guillemets dans l'autre), ces études essaient de déterminer la valeur des séquences concernées et la perception des nuances de sens par des lecteurs non experts. La confrontation de plusieurs points de vue est à cet égard riche en enseignements : elle permet de repérer convergences et divergences, de mettre au jour les choix d'annotation et éventuellement de trancher, si le but est d'obtenir une annotation aussi homogène que possible.

Du côté du traitement automatique des langues (TAL), le niveau discursif est pris en compte à travers des tâches telles que la segmentation thématique, qui oblige à entrer à l'intérieur du document pour en identifier les principaux thèmes (voir Hearst 1992 ou 
Ferret, 2006 parmi de nombreuses autres références) et pour repérer les changements de thème. Si les approches sont très informatisées et assez pauvres linguistiquement (les systèmes s'attachent généralement à repérer des répétitions de mots et des cooccurrences, avec ou sans ressources extérieures), elles montrent bien que la notion de cohérence est marquée par un ensemble d'éléments hétérogènes qu'il faut pondérer et prendre en compte simultanément. Des travaux récents ont montré la nécessité d'aller au-delà des mots (Valette 2004), voire de prendre en compte la structure des documents (Luc et Virbel, 2001; Péry-Woodley et Scott, 2006) afin de dépasser l'opposition entre approches fondées théoriquement et approches fondées sur les données (Biber et al., 2007). Quoi qu'il en soit, l'évaluation de tels systèmes est difficile: les notions de cohérence textuelle et de changement thématique ne sont pas évidentes et, de fait, il existe une grande variation d'un humain à l'autre (ceci est évalué en prenant en compte le taux d'accord inter-annotateur qui peut être calculé automatiquement).

Au-delà, les nombreuses campagnes d'évaluation en TAL posent des problèmes en apparence plus éloignés mais en fait tout à fait pertinents pour notre propos. L'extraction d'information (MUC-6, 1995) vise par exemple à extraire des événements d'un type particulier (par ex. des rachats d'entreprises, des interactions géniques, etc.). Afin d'évaluer les systèmes, des références (gold standard) sont mises au point manuellement, par annotation des données. Cette annotation est difficile car on a finalement assez rarement affaire à des événements simples et bien identifiés. Le plus souvent, les modalités, les négations et les circonstants brouillent le propos au point de rendre incertaine la décision à prendre quant à l'annotation d'une séquence donnée. À l 'instar de l'annotation de segments à visées pragmatiques, l'annotation des événements est difficile car l'objet visé ne correspond pas à une forme bien définie mais exige une part d'interprétation.

21 Il existe donc de nombreuses situations où le repérage des occurrences du phénomène est problématique. Les études montrent souvent des cas centraux, prototypiques, faciles à identifier et d'autres plus éloignés, moins facilement caractérisables. Une expérience a été menée récemment à l'Université de Pennsylvanie afin de produire une base de données textuelle enrichie d'informations discursives (la Discourse Penn Treebank, Miltsakaki et al., 2008). Quand la relation discursive à annoter concerne un pronom ou un connecteur, le taux d'accord entre annotateurs est satisfaisant. À l'inverse, quand l'annotation porte sur des relations implicites entre séquences textuelles ("implicit connectives"), le taux d'accord chute (les auteurs rapportent un accord de $72 \%$ pour les connecteurs implicites, au lieu de $90 \%$ pour les autres types de relations).

Il est donc nécessaire de voir sur quelle base peuvent être définies des règles d'annotation afin de maximiser l'accord entre annotateurs tout en s'assurant de bien prendre en compte la diversité des cas et des situations en corpus. Notre étude se situe dans cette lignée. Elle vise à caractériser certains segments à partir d'un faisceau d'éléments que l'on souhaite déterminer à partir des données elles-mêmes. Pour ce faire, nous nous sommes attachés à l'analyse des textes procéduraux. 


\section{Les procédures : problèmes spécifiques de définition et de repérage}

(quelle est la visée de ces textes? Comment sont-ils pris en compte?), linguistiques (quelles sont les caractéristiques de ces textes?) ou à des fins de traitement automatique (comment reconnaître les zones procédurales dans les textes ?). La plupart de ces études, sur lesquelles nous revenons dans ce qui suit, partent d'un ensemble de textes défini $a$ priori comme étant procéduraux : recettes, guides de montage, etc. Peu s'interrogent sur la notion même de procéduralité, ses contours et ses limites, même si certains comme Virbel (1997) partent d'une palette large de textes et essaient d'en cerner les spécificités. Malgré tout, le flou qui entoure la notion rend son étude complexe, et ce aussi en partie parce que les contours de l'objet d'étude fluctuent selon la discipline impliquée.

Les psycholinguistes se sont intéressés aussi bien à la production qu'à l'interprétation des textes procéduraux (Fayol, 1997). Ces textes ont retenu leur attention car ils expriment des façons d'agir ou de réaliser des tâches variées. De ce point de vue, le choix des textes est guidé par l'objectif : les psychologues doivent avoir accès à des conditions d'exécution et de mise en pratique du texte pour en étudier l'impact en situation réelle. Ainsi, L. Heurley et $\mathrm{F}$. Ganier se sont intéressés à l'analyse des textes procéduraux d'un point de vue psycho-cognitif, en essayant de mettre en regard structure des textes et traitements cognitifs associés (Heurley et Ganier, 2006). Heurley (2001) part du constat que la structure des procédures, leur emplacement et leur mise en langue sont importants, notamment pour la bonne exécution de la tâche visée. La façon dont le texte est structuré a par exemple une influence sur le temps d'exécution ou sur le respect plus ou moins strict de la procédure. L'étude porte sur des textes considérés comme procéduraux mais les auteurs ne posent pas en tant que telle la question de la délimitation du corpus d'étude. Tout texte pouvant déboucher sur une réalisation pratique, observable de manière objective, est 'bon à prendre'. De fait, une large portion de textes est ignorée, comme les conseils, qui ne débouchent pas directement sur des actions à réaliser.

Les approches linguistiques des textes procéduraux (Virbel, 1997 ; Adam, 2001) montrent l'hétérogénéité des types de textes concernés : la diversité de leur finalité, la façon dont ils mettent en langue la notion de procédure et la grande importance de la dimension illocutoire au sein de ces documents. De ce fait, ces approches ont des affinités avec notre façon de poser le problème. Adam (2001) montre qu'un texte procédural (comme une recette de cuisine) ne peut être assimilé à un récit, contrairement à ce qu'ont proposé divers auteurs (e.g. Greimas, 1983). En effet, la présence de verbes d'action et l'importance de la temporalité ne suffisent pas à identifier les textes procéduraux. Ceuxci sont avant tout associés à des situations particulières, ils sont essentiellement factuels et tournés vers un but précis entraînant une modification de l'état du monde réel (et non fictionnel). En conséquence, ces textes, aussi divers soient-ils, comportent un certain nombre de marqueurs linguistiques de la position illocutoire de l'auteur du texte : verbes d'action, d'ordre et de conseil, etc. L'analyse de la position relative de l'auteur, du récepteur et la nature du message évoqué est dès lors primordiale mais il ne s'agit pas d'un critère suffisant pour définir la notion de procéduralité.

26 C'est pourquoi Virbel (1997) a proposé une caractérisation des textes procéduraux (qu'il appelle textes de consignes) fondée sur la théorie des actes de langage. Son article se 
décompose en deux parties : dans un premier temps, il énumère plusieurs facteurs ayant une influence sur le type de consigne en jeu. Dans un deuxième temps, il détaille les forces illocutoires à l'œuvre dans les consignes. Virbel souligne d'abord que les consignes recouvrent un ensemble d'éléments hétérogènes qui tous participent à la bonne compréhension du texte. Ces éléments (statut de l'auteur de la consigne, façon dont celleci est rédigée, position de la consigne, etc.) ont une incidence sur la façon d'exprimer la consigne, sur le contenu linguistique et sur la force illocutoire employée. Ainsi, un médecin qui s'adresse à ses pairs ne peut pas être trop directif et doit justifier les choix thérapeutiques recommandés. Une notice de bricolage ou une recette de cuisine, à l'inverse, décrivent une liste d'actions et laissent en principe peu de place à la discussion ou à la justification (mais même là, une action qui risque de paraître surprenante pour un lecteur moyen devra être justifiée). L'étude de Virbel donne donc des clés pour mieux appréhender la diversité de l'objet d'étude, à défaut d'en identifier clairement le périmètre.

Quelques études en traitement automatique des langues se sont également intéressées à la notion de procédure (Yin et Power, 2006; Takechi et al., 2003). Il s'agit, essentiellement dans le cadre de systèmes de questions-réponses, d'identifier des zones susceptibles de répondre à des questions en « comment». Yin et Power (2006) essaient de caractériser le document dans son ensemble comme étant procédural ou non, mais obtiennent des résultats mitigés. Takechi et al. (2003) obtiennent de meilleurs résultats mais limitent leur étude au repérage de listes dans les documents, en s'appuyant sur le codage HTML. Tout ce qui n'est pas explicitement dans une structure de liste au niveau du codage HTML n'est pas analysé. Malgré ses limites, cette dernière approche montre bien que l'on ne peut considérer le document comme un bloc mais qu'il est nécessaire de repérer à l'intérieur du texte des zones pouvant être qualifiées de procédurales. Les auteurs montrent en outre la nécessité d'appuyer l'analyse sur divers éléments (système typo-dispositionnel, marquage linguistique, etc.). Ces études sont toutefois très limitatives et en définitive assez peu intéressantes pour notre propos : elles portent en effet sur des séquences bien identifiées par des marques explicites. De nombreuses zones procédurales sont volontairement laissées de côté, faute d'indices clairs pour les repérer.

Les différents travaux que l'on vient d'examiner manifestent une relation étroite entre l'objectif de recherche, la définition de l'objet de la recherche et les données considérées. Pour une étude psychologique portant sur la réception des consignes, sont procéduraux les textes qui peuvent être incarnés dans un « faire » observable (construction d'un objet, réalisation d'un schéma, utilisation d'un artefact...). Pour une analyse linguistique descriptive, la définition peut être plus large, c'est ainsi, par exemple, que J.-M. Adam (2001) étend la catégorie jusqu'aux horoscopes en ce qu'ils comportent des potentialités d'action. Pour les applications de type "Questions-Réponses", seront avant tout considérés les textes qui comportent des marques formelles explicites de consignes ou d'instructions. La conception même de ce que sont une procédure et un texte procédural peuvent donc au bout du compte constituer un biais considérable pour les résultats ultérieurs de l'analyse. La méthode que nous exposons maintenant tente de réduire ce biais potentiel. 


\section{Méthode et résultats}

Rappelons que cet article se focalise sur une méthodologie de recueil de données pour une analyse en corpus de phénomènes relevant du niveau discursif, et non sur la constitution du corpus lui-même. Il faut cependant rassembler un corpus d'étude, pour avoir matière à description de séquences. Afin de ne pas introduire nos propres préjugés sur ce qui est procédural et ne l'est pas, nous avons pris le parti de recourir à un jugement « extérieur » pour le choix des textes. Ce choix et le corpus de textes qui en découle sont présentés en détail dans la section 4.1.

Une fois les textes réunis, le recueil des données met en œuvre un protocole d'annotation « humaine et manuelle » des séquences procédurales, appuyé sur la définition proposée section 4.2 et explicité section 4.3. L'annotation produit deux types de données susceptibles de nourrir la réflexion: d'une part, dans les textes mêmes, un ensemble de séquences suscitant un jugement unanime des annotateurs et de séquences suscitant des jugements divergents; d'autre part, une mesure chiffrée du taux d'accord entre les annotateurs (section 4.4). Ces données autorisent un examen précis des segments textuels «accords » et " désaccords » entre annotateurs grâce auquel on peut mieux cerner les contours du phénomène étudié, en apprécier les exemplaires typiques et les zones floues. Ce retour sur les segments est détaillé section 5 .

\subsection{Constitution du corpus d'étude}

Afin d'être le moins limitatif possible et d'avoir une vision contrastée des faits linguistiques, nous avons choisi de collecter des textes dans deux domaines et genres textuels différents :

- des fiches mises à disposition sur Internet par un magazine «domestique » grand public, le site « Maison Facile »", accessibles sous des rubriques telles que 'Cuisiner' 'Bricoler' 'Décorer' 'Entretenir' 'Jardiner';

- des "Guides de bonne pratique médicale " élaborés par des comités de médecins à destination de médecins praticiens, en vue d'aider ces derniers à intégrer les avancées de la recherche médicale dans leur pratique.

Les premières font typiquement partie de ce que la littérature linguistique et psycholinguistique analyse généralement comme textes procéduraux. Ce sont les parangons de la catégorie, ceux qui en forment le cœur.

Les seconds sont des documents explicitement définis comme «recommandations professionnelles " à destination des médecins, ils ont d'ailleurs pour dénomination alternative "Recommandations pour la pratique clinique » (practice guidelines) et sont destinés à fournir « des propositions développées selon une méthode explicite pour aider le praticien et le patient à rechercher les soins les plus appropriés dans des circonstances cliniques données» (définition de la Haute Autorité de Santé). Rappelons que la recommandation figure précisément dans la typologie des consignes élaborée par Virbel (1997), ces documents entrent donc légitimement dans le champ de notre étude.

Même si nous sommes loin de la diversité souhaitable, le corpus couvre deux domaines, médical et « domestique » (au sens de " ce qui se rapporte à la maison»), et est constitué 
de deux genres différents, guides de bonne pratique et fiches explicatives. Cette dernière assertion sur les genres mérite un mot d'explication.

Si nous analysons les deux sous-ensembles du corpus comme relevant de genres différents, c'est en fonction d'une conception du genre qui relie celui-ci tout à la fois à une pratique sociale et à une visée illocutoire ${ }^{5}$. Comme nous l'avons indiqué ci-dessus, la visée des documents médicaux est de fournir aux médecins praticiens des recommandations pour améliorer leur pratique, mais ils ne constituent ni un «mode d'emploi » ni une "recette » stricto sensu. Le site «Maison facile » propose au contraire des fiches relativement courtes pour indiquer au lecteur comment s'y prendre pour tel aménagement, bricolage ou entretien dans la maison, ces fiches relèvent donc dans leur intention de la recette.

À l'étape de constitution du corpus, cette différence de genre et de domaine est une tentative de rupture contrôlée avec les sempiternels exemples et analyses fondés sur les recettes de cuisine ou les notices de montage de meubles, qui constituent certes un exemple typique d'action guidée, mais ne sont en fait pas les seuls exemplaires de procédures que l'on puisse envisager.

À partir de ces textes, pour la réflexion sur le recueil des occurrences, il s'agit de repérer les zones dans lesquelles des procédures sont décrites. Il convient donc, en premier lieu, de se doter d'une définition de ce qu'est une procédure; en second lieu, d'identifier les exemplaires de procédures à l'intérieur des textes, ce qui revient au bout du compte à produire un jugement d'adéquation d'une zone textuelle à la définition adoptée.

\subsection{Définition des procédures}

On a souligné dans l'introduction l'impossibilité d'asseoir une définition des procédures sur leurs caractéristiques linguistiques. L. Heurley (1997) donne du texte procédural une définition appuyée sur sa fonction principale.

Cette définition est reprise et précisée dans la suite de son travail par des considérations sur la nature des informations communiquées par ce type de textes:

«La notion de texte procédural [...] fait référence à tous les textes dont la fonction principale est de communiquer des procédures en vue d'une exécution ponctuelle (ex. : comment utiliser une consigne automatique, comment faire face à une situation d'incident, etc.) ou d'un apprentissage à long terme censé permettre l'acquisition d'un savoir-faire nouveau dans un domaine particulier [...]. Les textes procéduraux ont donc avant tout une fonction pragmatique [...]. Cette catégorie regroupe, notamment, les modes d'emploi, les notices explicatives, les manuels et les guides d'utilisation, les consignes de sécurité, les recettes de cuisine, les do-lists utilisés en aéronautique, etc. [...]

La première caractéristique des textes procéduraux est qu'ils communiquent à la fois des informations déclaratives et procédurales [...]. Les informations déclaratives correspondent à toutes les informations qui ne sont pas directement orientées vers l'action. Ces informations sont présentes dans les textes procéduraux comme dans toutes les autres catégories textuelles. [...] Les informations procédurales spécifient, quant à elles, des opérations ou des actions que l'utilisateur doit exécuter (instructions positives), ou au contraire, s'abstenir d'exécuter (instructions négatives).» (Heurley, $2001: 30-31$ )

Sans préjuger de la nature des informations qui se trouvent dans les textes procéduraux ${ }^{6}$, nous partons de la proposition réaffirmée qu'un segment procédural se définit par sa fonction principale, qui est de communiquer un ensemble d'actions à exécuter. 
41 On voit bien cependant le danger difficilement évitable de ces définitions : pour esquiver l'écueil d'une définition circulaire qui ferait intervenir des formes linguistiques alors même que ce sont celles-là que l'on veut découvrir, on bascule vers une définition qui se fonde sur la visée pragmatique principale du texte. Il est clair qu'intervient alors une part non négligeable de subjectivité. De fait, une telle définition ne peut constituer qu'un point de départ, elle doit être affinée par une tâche d'identification des segments textuels procéduraux. À cette fin, nous avons mis en œuvre une annotation manuelle reposant sur plusieurs annotateurs.

\subsection{Annotation manuelle des segments procéduraux}

42 L'objectif de l'annotation manuelle est de marquer les endroits où s'expriment des procédures - selon la définition indiquée précédemment - tout en mesurant la part de subjectivité impliquée dans cette tâche. L'annotation permet donc tout à la fois d'obtenir des chiffres quantifiant la convergence ou la divergence des jugements et de mettre en lumière les segments textuels qui suscitent consensus ou désaccord. Les annotateurs devaient simplement se prononcer sur le caractère procédural de chaque segment textuel, en encadrant les segments jugés procéduraux de balises nommées 'SeqProc' (en fait, une marque analogue à un surlignage). Il ne s'agissait pas d'une tâche de segmentation dans la mesure où cette annotation a pris pour base la division originelle du texte en sections titrées. Nous avons décidé de procéder ainsi essentiellement parce qu'une section est une unité textuelle dont les bornes sont données de façon fiable : une section commence avec un titre (parfois avec une simple numérotation, mais nous n'avons pas rencontré ce cas dans les documents du corpus) et se termine avec le titre suivant ou avec la fin du document. Ceci évite de se poser la question de savoir où commence et où finit le segment à annoter. Une telle décision n'est pas sans conséquences parfois indésirables, notamment l'amalgame des parties procédurales et non procédurales de sections hétérogènes, mais elle a eu pour mérite de ne pas rajouter une tâche de segmentation de textes à la question de l'appréciation de la " procéduralité ».

Le corpus Médecine a servi de "banc d'essai" avec une première phase d'annotation d'un petit échantillon de textes effectuée par deux annotateurs. Cette première phase a produit deux sortes de résultats. D'une part, nous avons recueilli une évaluation chiffrée du taux d'accord des annotateurs, d'autre part, à partir de la confrontation des jugements de chacun, nous avons pu établir un guide utilisé pour l'annotation du corpus Maisonfacile, ce qui n'a pas empêché les désaccords comme nous le verrons plus bas.

44 La seconde phase d'annotation a donc concerné le corpus MaisonFacile, en impliquant aussi deux annotateurs.

\subsection{Résultats chiffrés de l'annotation}

Comme l'annotation ne s'est pas déroulée dans les mêmes conditions pour les deux corpus, nous différencions les résultats. 


\subsubsection{Corpus Médecine}

Pour apprécier les résultats qui suivent et les conclusions que nous en tirons, il faut garder présent à l'esprit que l'annotation sur le corpus Médecine s'est effectuée sans guide et sans autre consigne que « repérer ce qui parait exprimer une procédure ». Cet appel à l'intuition avait précisément parmi ses objectifs d'estimer le partage ou non des intuitions ${ }^{7}$.

Le tableau 1 indique les résultats obtenus pour l'annotation. Dans ce tableau comme dans le tableau 2, on indique pour chacun des deux annotateurs le nombre de segments qu'il a jugés 'procédural' et le nombre de segments qu'il a jugés 'non procédural'. Les marges indiquent le total de chaque ligne et de chaque colonne, la case la plus extrême en bas à droite indique le total des totaux, c'est-à-dire le nombre total de segments (307 pour cette annotation). Les nombres en caractères gras signalent les accords de jugement (152 + $113=265$ ), les désaccords portant quant à eux sur 42 segments.

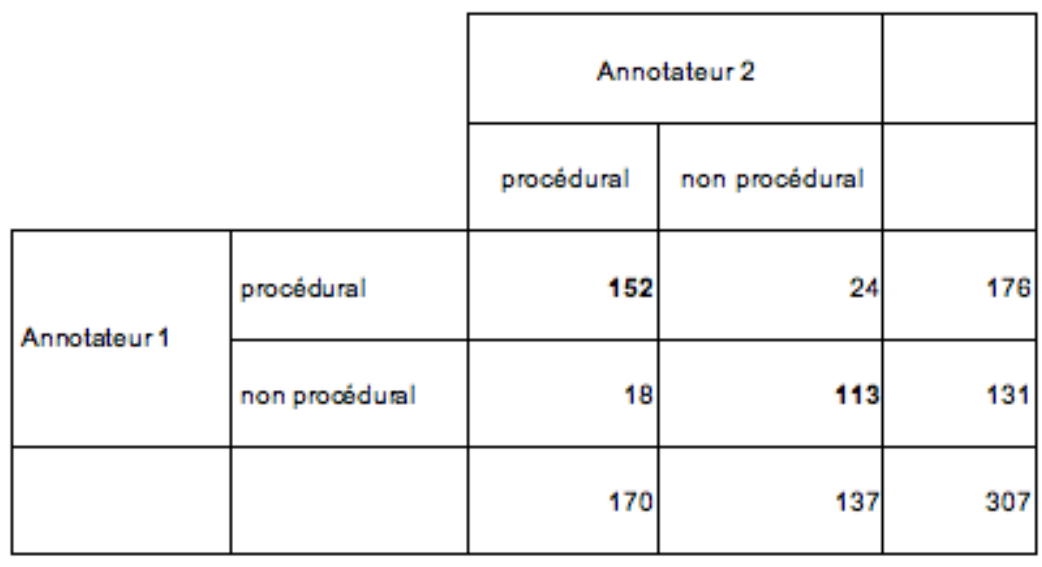

Tableau 1 : résultats de l'annotation du corpus Médecine (les cas d'accords se trouvent à Fintersection des lignes et des colonnes dont lintitulé est similaire, les autres cases figurent les cas de désaccord).

L'indice statistique Kappa est un bon moyen de mesurer le degré d'accord interannotateurs (Carletta, 1996). En appliquant la formule de calcul du Kappa ${ }^{8}$

$\mathrm{K}=(\mathrm{Po}-\mathrm{Pe}) / \mathrm{1}-\mathrm{Pe}$

on obtient :

$\mathrm{K}=(265 / 307)-0,5079 / 1-0,5079=0,72$

On considère généralement comme bonne une valeur de Kappa comprise entre 0,61 et 0,80 .

Cette première annotation a permis de cerner quelques difficultés liées à la tâche et à la prise de décision - comme par exemple l'appréciation de tournures impersonnelles du type "la patiente devra être informée ", équivalente à une injonction "informez la patiente " - qui ont été formalisées sous forme de conseils rédigés et transmis aux annotateurs du second corpus.

\subsubsection{Corpus MaisonFacile}

Pour le corpus Maisonfacile, précisons que l'un des deux annotateurs avait été impliqué dans la première série d'annotations. 
Comme on peut le lire dans la case inférieure droite du tableau 2, le nombre de segments à annoter est à peu près similaire (275), tandis que le nombre de désaccords est réduit de moitié $(3+17=20)$.

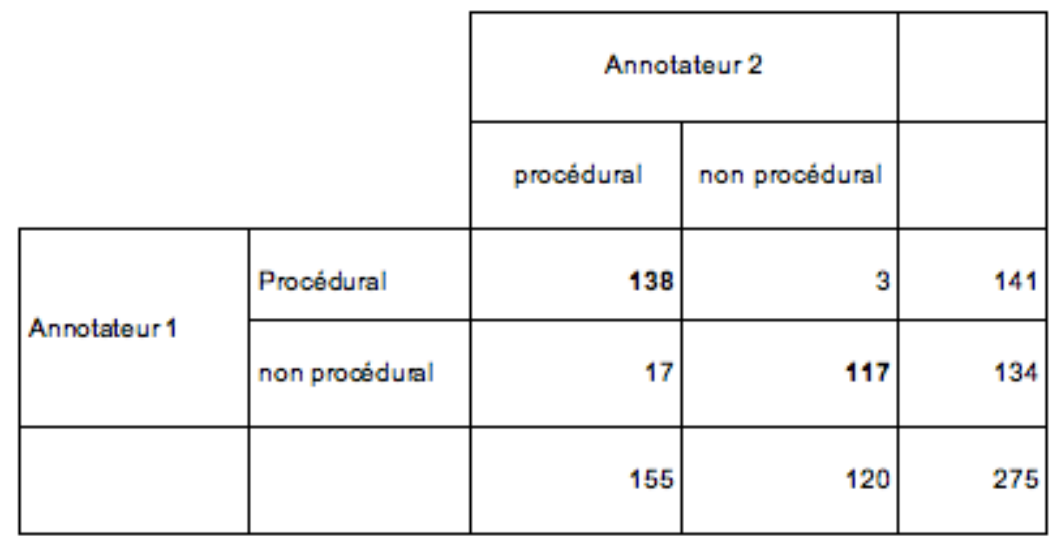

Tableau 2 : résultats de l'annotation du corpus MaisonFacile

Comme le laisse présager la lecture du tableau, l'indice Kappa manifeste un meilleur accord inter-annotateur :

$\mathrm{K}=(255 / 275)-0,5 / 1-0,5=0,85$

En effet, 0,85 est considéré comme signe d'un accord excellent.

\subsubsection{Commentaires sur l'annotation}

Les valeurs de kappa obtenues montrent que globalement les annotateurs sont plus souvent en accord que si cette décision était laissée au hasard, ce qui est somme toute rassurant: on peut alors espérer dégager des régularités à partir desquelles fixer les contours de l'objet « segment procédural ».

La simple prise en considération des chiffres d'accord et de désaccord permet déjà de conclure à l'existence d'un « noyau dur » de la procéduralité, c'est-à-dire les segments qui font l'unanimité des jugements indépendamment des textes, de leur genre et de leur domaine, indépendamment des annotateurs. À partir de là, une définition de la procéduralité peut être affinée en dégageant les caractéristiques de ce noyau dur. Complémentairement, il existe aussi une "zone limite " dont l'appartenance à la catégorie "procédure " est plus polémique. Il s'agit maintenant d'analyser plus précisément ces ensembles pour :

- déterminer si les segments d'un même sous-ensemble (unanimité vs désaccord) présentent des caractéristiques communes

- utiliser le classement des annotateurs pour affiner la définition de la procéduralité.

\section{Cerner la procéduralité grâce à l'annotation}

Au-delà des chiffres mesurant le taux d'accord, la tâche d'annotation manuelle procure une gradation quant à l'appréciation du caractère procédural. L'intérêt de savoir pour chaque segment s'il a fait l'objet d'un accord unanime ou de jugements divergents réside dans la mise en relation avec les moyens linguistiques observables dans ce segment. Les questions auxquelles on peut alors répondre sont « pour quelle(s) raison(s) ces segments 
font-ils l'unanimité / suscitent-ils un désaccord ? Peut-on dégager des types marqués par les procédés linguistiques employés?».

Dans cette section, nous mettons en évidence le fait que le jugement est fortement corrélé aux moyens linguistiques mobilisés. Pour le dire vite, plus le segment considéré comporte des éléments explicitement destinés à guider l'action, plus il fait l'objet de consensus et inversement, plus il use d'implicite, plus il fait l'objet de divergences.

Nous proposons une typologie des segments procéduraux qui s'appuie sur les procédés linguistiques observés. Certains types sont propres au corpus Médecine, leur description est alors précédée de [Méd], d'autres sont présents dans les deux corpus analysés.

\subsection{Accord parfait sur la procéduralité}

Commençons par les segments que les annotateurs se sont accordés à juger procéduraux. On observe 5 types de procédés linguistiques en ce cas.

- Procédures « prototypiques"

L'accord des annotateurs est parfait particulièrement lorsque les segments correspondent aux descriptions proposées dans les travaux sur les textes procéduraux et en manifestent les caractéristiques linguistiques et discursives : mention d'un but à atteindre, description d'actions à mettre en œuvre, indication de la temporalité de ces actions (séquences, parallèles...). En voici un exemple extrait du corpus Médecine ${ }^{9}$ :

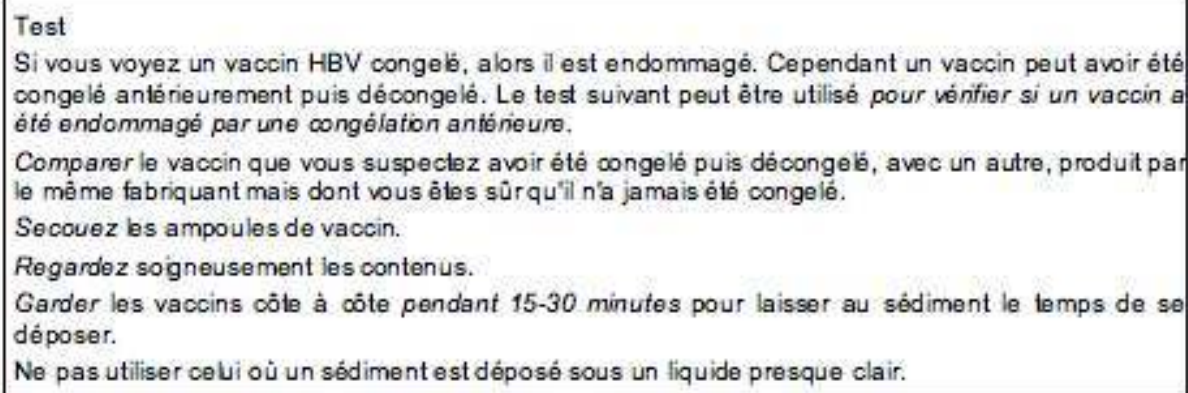

60 Sont ici exploités les ingrédients généralement mentionnés de l'expression des consignes : verbes à l'impératif ou à l'infinitif, énumération, successivité. Ils ont été largement décrits dans la littérature et marquent le type "Recette de cuisine ». Il n'est donc pas du tout étonnant de voir ce type faire l'unanimité : c'est le parangon de la catégorie.

\section{- Structure de cas}

61 Moins prototypiques mais aussi consensuels, des segments qui ne se contentent pas d'indiquer des actions à réaliser, mais qui différencient la procédure à mettre en œuvre en fonction de cas. Cette structure de cas - rarement mentionnée dans les études linguistiques (pas davantage dans les études psychologiques ou psycholinguistiques) sur les textes procéduraux - est particulièrement présente dans le domaine médical où le choix du traitement est déterminé par la pathologie du patient, comme on le voit cidessous. 


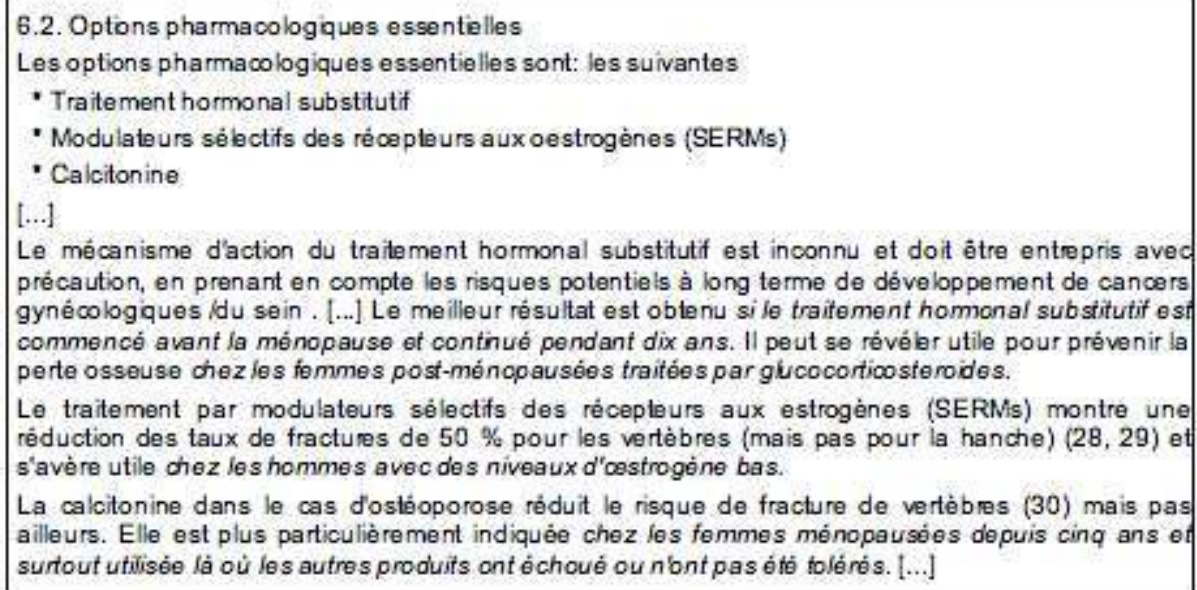

62 Cette structure ne se limite toutefois pas au domaine médical, elle est présente aussi dans le corpus Maisonfacile, qui couvre à l'occasion des questions relatives à la vie courante. Le texte suivant, qui explique "comment borner un terrain », examine successivement deux situations possibles, 'les voisins sont d'accord / les voisins ne sont pas d'accord', et indique le programme d'actions à exécuter dans chacune de ces situations.

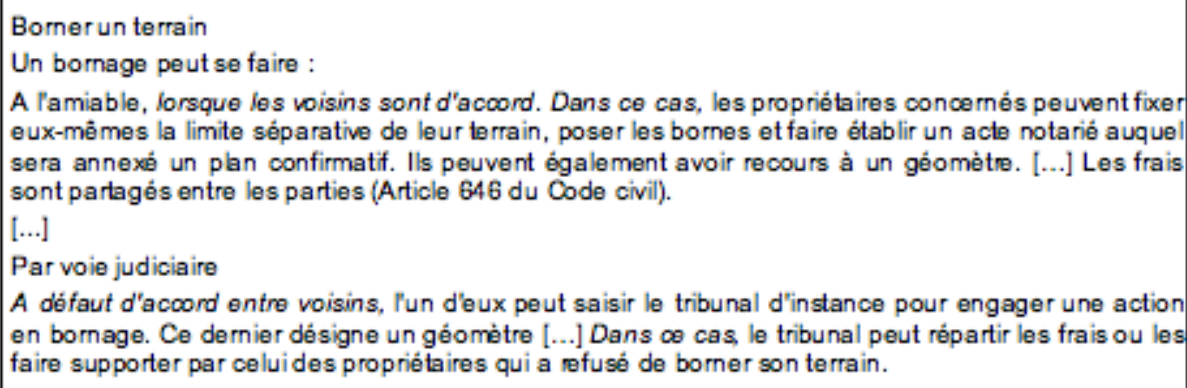

\section{- [Méd] Actions « désincarnées »}

63 Au contraire du premier type de cette section (le type « recette de cuisine »), le corpus Médecine use volontiers de formulations par lesquelles les actions «se réalisent d'ellesmêmes ", ce qui sur le plan linguistique se traduit par la quasi disparition de l'impératif ou de l'infinitif au profit de déverbaux, de formes passives ou impersonnelles.

Dans le segment qui suit, pas la moindre instruction explicitement adressée au médecin pour la réalisation de la manométrie œsophagienne, pourtant, au final, celle-ci est bel et bien décrite en tant que procédure à mettre en œuvre, ce qui a été reconnu par l'ensemble des annotateurs. 
Réalisation de rexamen.

$[\ldots]$

Les modalités de réalisatón de la manométrie oesophagienne font lobjet de pratiques assez homogènes [...]. Après introdudion de la sonde, de préférence par voie nasale, les onfices de perfusion sont tous placés dans la cavité gastrique. Ceci est vérifié par une inspiration profonde qu se traduit alors par une ascension de toutes les courbes de pression. Il est exceptionnel qu'une mise en place sous contrôle radioscopique ou endoscopique soit nécessaire. Le SiO, le corps de roesophage et le SSO sant ensuite explorés successivement.

- Sphincter inférieur de loesophage (SIO). La technique du retrait rapide ne pemettant pas fétude des relaxations ef nécessitant un appareillage spécifique n'est plus utilisée. Dans la configuraton minimale décrite plus haut, fétude du SIO séfiectue donc par le retrait lent de la sonde (paliers de 1 ou $1 / 2 \mathrm{~cm}$ ). Elle peut étre aussi effechuée avec des cathéters dont les onfices sant disposés de façon radiaire dans le mëme plan (ou à $1 \mathrm{~cm}$ les uns des autres). Bien que plus rapide, cette modalité nécessite une pus grande expérience de Pexaminateur. En tout cas, la fechnique de recueil des données doit être similaire à celle utilisée pour définir les nomes (1). Au moins 4 déglutitions effectuées avec un bolus d'eau de $5 \mathrm{~m}$ (déglutitions humides) sant nécessaires pour étudier la relaxation du SIO. Le passage de Pabdomen à la région thoracque est défini par le point d'inversion de pression.

- Corps de loesophage. Les modalités de létude du corps de loesophage sont fonction du type de sonde utilisée, et plus précisément, du nombre d'orifices perfusés, mais une exploration de
rensemble du corps de foesophage est toujours nécessaire $[. .$.

65 Les actions à réaliser sont parfois aussi signifiées par des lexèmes spécifiques, particulièrement dans le domaine médical. Dans l'extrait suivant, ce sont les termes cytoréduction et chimiothérapie qui indiquent au médecin quoi faire face à une carcinose péritonéale.

1. La carcinose péritonéale chez une femme
Le diagnostic de carcinome papillaire séreux (alors que les ovaires sont normaux) invite à traiter une
carcinose péritonéale de la même façon qu'un cancerde lovaire. [...]
Une cytoréduction chinurgicale optimale, suivie d'une chimiothérapie associant un dénivé du platine
permet d'espérer une survie de lordre de 24 mois, $\infty$ mme dans un cancer de lovaire.

66 L'annotation d'un tel segment n'a pas posé problème, on peut supposer que les annotateurs se sont appuyés sur la compositionnalité des termes pour reconnaitre des noms d'actions.

\section{- Injonction}

Les annotateurs ont été sensibles à l'expression d'injonctions. Dans le corpus Médecine, l'impératif est peu utilisé, toutefois une forte injonctivité s'exprime par un lexique de l'obligation et de la nécessité. Ce dernier indique que le segment considéré remplit une fonction de consigne et c'est sur celui-ci que s'opère la convergence des annotateurs.

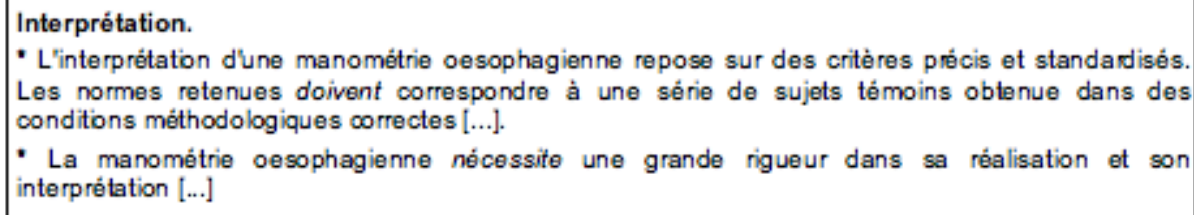

\section{- [Méd] Description de normes}

68 Dans le corpus médical, les consignes peuvent porter moins sur des actions à effectuer que sur une organisation ou un matériel. Nous avons défini ce type comme « description de normes ", car ces segments décrivent non pas «ce qu'il faut faire » mais « ce qui doit être » et "comment cela doit être », c'est-à-dire des caractéristiques impératives d'un matériel ou d'une organisation (par exemple, la composition d'une équipe de soignants). 


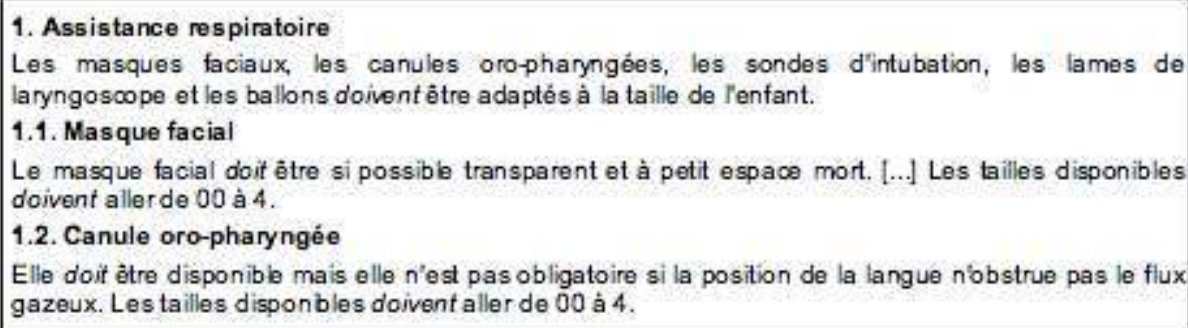

Les annotateurs ont tous jugé cet extrait comme une succession de segments procéduraux, sensibles à l'expression de la nécessité (répétition du verbe devoir). Mais en même temps, on va le voir immédiatement, certains autres segments que nous définissons aussi comme «descriptions de normes» sont la principale source de désaccord entre les annotateurs.

\subsection{Désaccords}

Reprécisons que nous rangeons ici tous les segments pour lesquels le jugement des annotateurs diverge : procédural pour les uns, non procédural pour les autres ${ }^{10}$.

- [Méd] Description de normes

71 Les segments dont il était question dans la section précédente comportent des marques lexicales d'injonctivité et ce sont ces dernières qui aident à interpréter le segment comme exprimant une obligation d'agir: si l'on écrit «la canule oro-pharyngée doit être disponible », le lecteur peut en inférer « vous devez rendre la canule disponible... ».

72 Des jugements contradictoires surgissent à propos d'autres segments qui à première vue entrent dans ce type de la description de normes (tel que nous l'avons défini ci-dessus) sans toutefois employer de formes spécifiques de la consigne.

Dans le but de contribuer à une sécurité optimale du patient anesthésié, la Société française d'anesthésie et de réanimation (SFAR) fait les recommandations suivantes concemant Fapparei d'anesthésie et sa vérification avant utilisation. Il s'agit de recommandations minimales, à adapter au type d'appareil employé. Certaines situations d'urgence peuvent ne permettre qu'une vérification partielle de lappareil.

Si au moment de la publication de ces recommandations, un appareil d’anesthésie déä en fonction ne leur était pas conforme, la SFAR recommande au médecin anesthésiste-réanimateur de réaliser avec les responsables administratifs de fétablissement un plan pemettant à feme la mise en ceuvre des dispositions du présent document.

Ces recommandations, établies en fonction des données actuelles de la science et des normes. circulaires et textes réglementaires, seront soumises à une révision périodique.

1. Appareil d'anesthésie

L'appareil d'anesthésie permet l'administration d'O2 et d'agents anesthésiques par inhalation, ou éventuellement d'un mélange $\mathrm{O} 2$-air additionné ou non de vapeur anesthésique halogénée. Il permet la ventilation spontanée, manuelle et mécanique.

Un appareil complet incut un système (ou module) d'alimentation en gaz frais, un ou deux systèmes (ou circuits) anesthésiques, un ventilateur, un système antipollution (figure 1). Par ailleurs il est équipé de moniteurs et d'un aspirateur réservé à lusage anesthésique.

Seuls des appareils d'anesthésie homologués sont utilisés. Quand lappareil est constitué d'éléments homologués provenant de constructeurs différents, ils doivent être mutuellement compatibles et la cohérence de l'ensemble doit être vérifiée.

73 Le désaccord porte sur la section 1. Certains annotateurs, se fiant au fait qu'elle fait partie de recommandations et qu'elle décrit ce qui doit être en indiquant explicitement que cela doit être (les passages que nous avons mis en italique dans le début du document), l'ont considérée comme constituant des instructions. Les autres l'ont considérée comme uniquement descriptive, sans valeur injonctive. Cette dernière position se justifie d'autant plus que le début du document, s'il indique qu'il y aura bien quelque chose à 
faire dans les cas de non conformité (« réaliser un plan permettant à terme la mise en oeuvre des dispositions du présent document »), ne donne pas de programme d'actions clair pour chaque «infraction" à la recommandation - concrètement, que faire si l'appareil n'est PAS «équipé de moniteurs et d'un aspirateur réservé à l'usage anesthésique »?

De ce point de vue, on peut considérer que les descriptions de normes n'entrent pas exactement dans notre définition des procédures (cf. § 4.2). Il est important de souligner néanmoins que lorsque leur expression fait appel au lexique de l'obligation et de la nécessité, elles ont été jugées comme instructions.

\section{- Segments hétérogènes}

Un autre type de désaccord est lié à l'hétérogénéité de la section. Conformément à ce qu'indique L. Heurley en 2001 (cf. $\$ 4.2$ ), certains segments communiquent à la fois des informations déclaratives, généralement des données factuelles, et des instructions (en italique dans l'extrait suivant).

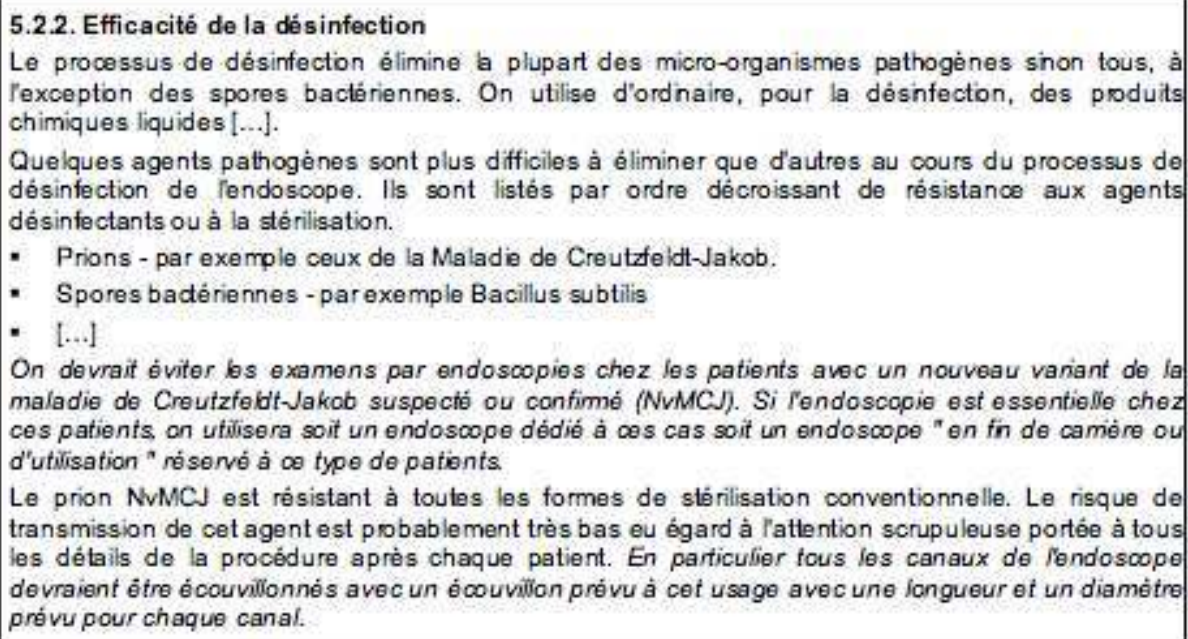

La variation vient de l'appréciation des statuts respectifs de chacun de ces types d'informations au sein de la section et partant, du statut global de la section. Si on interprète les morceaux factuels-descriptifs comme étant là pour soutenir les instructions, la section est jugée procédurale. Mais si on considère que l'intention première n'est pas centrée sur les actions et que les instructions sont uniquement mentionnées comme conséquence des informations factuelles qui sont énoncées et dont la communication est le réel objet de la section (ce qui dans ce cas précis est corroboré par le titre de section), alors la section n'est pas jugée procédurale.

Ce type de désaccord est en partie lié aux modalités de l'annotation (explicitées section 4.3) : nous avons décidé d'utiliser la segmentation «naturelle» du texte en sections, ce qui interdisait aux annotateurs de délimiter eux-mêmes ce qu'ils considéreraient comme procédural ou non procédural à l'intérieur du texte. En ce sens, on peut considérer que si nous laissions les annotateurs tracer les frontières des segments, cette catégorie de désaccord disparaitrait. Mais en fait, elle se déplacerait sur un autre point : la frontière elle-même du segment. Et quoiqu'il en soit, il est très probable que l'on retrouverait tout de même des « mixtes » de factuel et de procédural. 


\subsection{Retour sur la procéduralité}

78 Notre méthode d'annotation des textes a permis de faire le point sur la notion de procéduralité. Il en ressort que, même si l'interprétation reste déterminante pour juger qu'un texte a ou n'a pas pour fonction de communiquer un ensemble d'actions à effectuer, un noyau dur de segments textuels ne laisse place à aucune divergence. À côté de ceux-là, il y a des segments qui se situent à la marge de la catégorie, plus ou moins proches du centre selon le taux d'accord qu'ils suscitent.

79 L'analyse sous forme de typologie de l'ensemble de ces segments permet de dégager des éléments linguistiques décisifs pour les annotateurs. Ces éléments jouent le rôle de marqueurs et peuvent être enregistrés dans des guides d'annotation: ils fournissent une base explicite au consensus que l'on cherche à dégager. Notons toutefois que ces marqueurs eux-mêmes sont ambigus et dépendent étroitement du contexte dans lequel ils apparaissent. Il serait sans doute profitable, dans un travail qui se focaliserait plus précisément sur ces éléments linguistiques et sur leur rôle dans le marquage de la procéduralité, d'étudier spécifiquement leurs combinaisons, leurs interactions et leur « mise en texte». C'est assurément dans des configurations textuelles spécifiques que réside leur pouvoir d'indice de procéduralité.

80 Sans entrer toutefois dans le détail, mais pour récapituler ces éléments linguistiques et montrer qu'ils ne sont nullement spécialisés dans l'expression des procédures, indiquons que les segments procéduraux font usage de :

- Phraséologie propre au domaine

81 Par exemple, L'échographie est l'examen diagnostique de référence permet de signifier qu'il faut pratiquer une échographie, de même Ce médicament est reconnu comme efficace indique de prescrire ou d'inclure dans les hypothèses de prescription le médicament en question.

- Formes passives

D'une façon très similaire, nombre de recommandations sont exprimées indirectement, par exemple En cours d'anesthésie la position du patient est surveillée, en particulier les points d'appui ou Le traitement adjuvant est adapté à l'âge de la patiente.

- Marques d'injonction

83 À côté du classique modal devoir, les formes de l'injonction varient en gradation : cela va de la simple indication de préférence, qui semble laisser le choix au lecteur de s'y conformer ou de s'y soustraire, comme il est préférable de, à la prescription la plus impérative, comme il faut, sans oublier les mêmes formulations accompagnées de négation - les instructions négatives mentionnées par L. Heurley (cf. § 4.2) et que l'on retrouve abondamment dans les textes de médecine où il est parfois aussi important de ne pas faire que de faire, mais aussi dans le corpus Maisonfacile.

- Marques de condition

La place essentielle que tiennent les structures de cas dans le domaine médical conduit à l'emploi de marques de conditions telles que si, en cas de, dans le cas de, au cas où, dans la situation, lorsque, etc.

Il est remarquable que toutes les formes linguistiques que nous venons d'évoquer ne sont pas procédurales par nature. Elles remplissent ce rôle dans la mesure où le texte est perçu par le lecteur comme visant la communication d'un programme d'actions. Plus la visée 
pragmatique du texte (ou du segment de texte) est évidente pour le lecteur, plus les éléments linguistiques se voient attribuer ce rôle de support de cette visée pragmatique. On retrouve là un phénomène analogue à celui des marques de cohésion : ce ne sont pas les marques de cohésion qui font un discours cohérent mais c'est le fait que le lecteur y projette une certaine cohérence (Charolles, 1994).

De même, cette dimension interprétative explique les flottements observés lors de l'annotation et montre clairement que certains segments textuels sont "prototypiques " (au sens de Kleiber, 1990) tandis que d'autres se situent à la marge, à la périphérie de la catégorie, celle-ci ayant au bout du compte des frontières floues. Seule une annotation « à plusieurs mains » permet de le saisir en mettant en évidence les différences de jugement des annotateurs.

\section{Conclusion}

87 Notre expérimentation dégage des éléments de méthode qui vont au-delà de l'étude de la procéduralité. En effet, pour nombre d'études « linguistico-pragmatiques » en corpus, se pose la question de la définition de l'objet sur lequel on travaille. Même lorsque le linguiste dispose d'une définition des caractéristiques et propriétés de l'objet, la collecte des occurrences à analyser conduit souvent à des choix dans lesquels se révèle la part d'interprétation et de subjectivité nécessairement mobilisée lors de l'analyse des phénomènes discursifs. Des travaux sur les cadres de discours (Bilhaut, 2006) ou sur certaines anaphores (Landragin, 2007) mettent bien en évidence la difficulté d'opérationnaliser une définition sur des textes authentiques. De ce fait, le linguiste se voit souvent reprocher de présenter des données que sa propre interprétation seule a jugées pertinentes, et de tirer des conclusions de données incomplètes et/ou contestables.

88 La « multi-annotation » apporte plusieurs bénéfices. D’un côté, au fur et à mesure que des textes sont examinés, la définition du phénomène ou de l'objet se précise, ou tout au moins se précisent les aspects par lesquels peut s'élaborer une définition opératoire. De l'autre côté, les occurrences peuvent être classées en fonction d'un gradient de jugement : unanimité, presque unanimité, désaccord léger, désaccord total, etc. Enfin on peut apprécier le degré de convergence sur le phénomène dans son ensemble, par exemple à l'aide d'une mesure quantitative comme l'indice Kappa.

89 Au bout du compte, nous voulons argumenter pour une annotation ou tout du moins une phase de confrontation de jugements d'analystes divers non comme un préalable à une recherche, mais comme un moment essentiel de celle-ci. Le travail sur corpus comporte une étape de recueil des données qui est trop souvent passée sous silence ou présentée comme non problématique, en tout cas non problématisée. Or, qu'on le veuille ou non, qu'on l'explicite ou non dans la présentation des résultats, ce recueil de données implique une première construction non neutre de l'objet d'analyse. D'une certaine manière, le linguiste y projette un début de représentation. Dans notre étude, cette première projection a été mise à l'épreuve lors de la première phase d'annotation, sans guide. Celleci était moins destinée à cerner les occurrences dans les textes qu'à mettre en évidence la façon dont chaque annotateur se représentait le phénomène. Le guide d'annotation qui en est issu est en quelque sorte le produit du passage de conceptions et de stratégies individuelles à la construction stabilisée de l'objet d'étude. À partir de là peut s'opérer le recueil des occurrences pour la suite de l'analyse linguistique. 


\section{BIBLIOGRAPHIE}

Adam J.-M. (2001), « Entre conseil et consigne : les genres de l'incitation à l'action », Pratiques, n

-111/112, décembre 2001, pp. 7-38

Biber D., Connor U., et Upton T.A. (2007) Discourse on the move: using corpus analysis to describe discourse structure, Amsterdam, John Benjamins Publishing Company.

Bilhaut F. (2006). Analyse automatique de structures thématiques discursives - Application à la recherche d'information. Thèse de doctorat. Université de Caen.

Carletta J. (1996), « Assessing agreement on classification tasks : the Kappa statistic », Computational Linguistics 22(2), pp. 5-24.

Charolles, Michel (1994). « Cohésion, cohérence et pertinence du discours ». Travaux de linguistique 29, pp. 125-151.

Fayol M. (1997), Des idées au texte : psychologie cognitive de la production verbale, orale et écrite. Paris, Presses Universitaires de France.

Ferret 0. (2006) « Approches endogène et exogène pour améliorer la segmentation thématique de documents »; Traitement Automatique des Langues (TAL), vol. 47(2), pp. 111-135.

Greimas A. (1983), Du Sens II, essais sémiotiques. Paris, Le Seuil.

Hearst M. A. (1992), "Automatic acquisition of hyponyms from large text corpora", 14th International Conference on Computational Linguistics (COLING'92), pp. 539-545, Nantes.

Heurley L et Ganier F. (2006), « L'utilisation des textes procéduraux : Lecture, compréhension et exécution d'instructions écrites ». Intellectica. 44/2, pp. 45-62.

Heurley L. (1997), « Vers une définition du concept de texte procédural : le point de vue de la psycholinguistique », Les Cahiers du Français Contemporain 4, pp. 109-133.

Heurley L. (2001), « Compréhension et utilisation de textes procéduraux : l'effet de l'ordre de mention des informations ", Revue Française de Linguistique Appliquée 2001/2, Volume VI, pp. 29-46.

Kleiber, Georges (1990). La sémantique du prototype. Paris : P.U.F. - Linguistique nouvelle.

Landragin F. (2007), « L'anaphore à antécédent flou : une caractérisation et ses conséquences sur l'annotation des relations anaphoriques ", Journée d'étude de l'ATALA sur la résolution des anaphores, Paris : France. Consultable en ligne à http://halshs.archives-ouvertes.fr/docs/00/15/48/60/ PDF/07_ATALA.pdf,[consulté le 15 septembre 2010].

Leech G., McEnery T., \& Wynne M. (1997). “Further levels of annotation”. In R. Garside, G. Leech, \& A. McEnery (Eds.), Corpus Annotation: Linguistic Information from Computer Text Corpora. London : Addison Wesley, pp. 85-101.

Leroy S. (2004). « Extraire sur patrons : allers et retours entre analyse linguistique et repérage automatique », Revue Française de Linguistique Appliquée 2004/1 IX, pp. 25-43.

Luc C. et Virbel J. (2001). « Le modèle d'architecture textuelle Fondements et expérimentation », Verbum, vol. 23(1), pp. 103-123. 
Miltsakaki E., Robaldo L., Lee A., and Joshi A. (2008). “Sense Annotation in the Penn Discourse Treebank". In Proceedings of the 9th International Conference on Intelligent Text Processing and Computational Linguistics, Haifa, Israel.

MUC-6 (1995). Proceedings of the sixth Message Understanding. Conference (MUC-6), Morgan Kaufmann, San Francisco

Péry-Woodley M.-P. (2001), « Modes d'organisation et de signalisation dans des textes procéduraux », Langages 141, pp. 28-46.

Péry-Woodley M.-P. et Scott, D. (2006), « Computational Approaches to Discourse and Document Processing ", TAL, 47/2, pp. 7-19.

Rinck F. et Tutin A. (2007). «Annoter la polyphonie dans les textes : le cas des passages entre guillemets », Corpus [En ligne] 6 | décembre 2007, mis en ligne le 02 juillet 2008, Consulté le 15 septembre 2010. URL: http://corpus.revues.org/index1102.html

Stede M., Wiebe J., Hajicova E., Reese B., Teufel S., Webber B., Wilson T. (2007), “Discourse annotation working group report". In: Proc. of the Linguistic Annotation Workshop (LAW) at ACL 07, Prague, 2007.

Takechi M., Tokunaga T., Matsumoto Y. \& Tanaka H. (2003), "Feature Selection in Categorizing Procedural Expressions", The Sixth International Workshop on Information Retrieval with Asian Languages (IRAL2003), pp. 49-56.

Valette M. (2004) « Sémantique interprétative appliquée à la détection automatique de contenus illicites Approches Sémantiques du Document Numérique », 7e Colloque International sur le Document Electronique (CIDE), pp. 215-230.

Virbel J. (1997), «Contributions de la théorie des actes de discours à une taxinomie des consignes ", Cognition, Discours Procédural, Action (volume II). Actes de l'Atelier de Prescot « Texte et Communication », J. Virbel, J.-M. Cellier, J.-L. Nespoulous (eds.), Prescot, pp. 1-44.

Yin L. \& Power R. (2006), "Adapting the Naive Bayes Classifier to Rank Procedural Texts", European Conference on Information Retrieval (ECIR 2006), pp. 179-190.

\section{NOTES}

1. Par exemple, le repérage d'une antonomase (Leroy, 2004).

2. Nous n'ambitionnons pas, à ce stade et dans cette étude, de poser les bases de la constitution d'un corpus de textes procéduraux ; cette question serait en quelque sorte une étape suivante, dans une démarche qui est: d'abord cerner les contours du phénomène puis, à partir de là, déterminer les types et genres de textes susceptibles de présenter le phénomène.

3. Il existe sinon des cas d'erreurs manifestes de jugements qui sont rapidement résolus lors de la mise en regard des différentes annotations.

4. http://www.maison-facile.com/ [site consulté en septembre 2010]

5. Cf. notre introduction à la journée de l'ATALA (Association pour le Traitement Automatique des Langues) «Typologies de textes pour le traitement automatique », http://www.atala.org/ IMG/zip/presentations.zip [consulté le 10 septembre 2010].

6. La double nature indiquée peut éventuellement constituer un point que l'analyse linguistique réfutera ou confirmera.

7. On n'a malheureusement pas pu compléter l'étude par une nouvelle phase d'annotation pour mesurer l'accord sur le même corpus après confrontation des premières annotations et analyse des cas problématiques. Toutefois, un petit nombre de textes a été soumis au jugement de deux 
autres annotateurs et les chiffres obtenus sur ce petit nombre confortent ceux qui sont présentés ici.

8. Po étant la proportion d'accord observée et Pe étant la proportion d'accord aléatoire, c'est-àdire la proportion d'accord qui serait obtenue uniquement par chance ou par hasard.

9. Dans les extraits qui illustrent les types, nous mettons en italique les éléments linguistiques sur lesquels nous voulons attirer l'attention.

10. Quatre annotateurs se sont prononcés sur ces segments.

\section{RÉSUMÉS}

Cet article porte sur des problèmes d'analyse en corpus de structures discursives, en partant de l'exemple de la procéduralité. Quand l'objet d'étude ne porte pas sur une forme particulière, il est difficile de recueillir les données à analyser sans idée préconçue, c'est-à-dire sans biaiser a priori les résultats. L'article propose une méthode permettant de résoudre en partie ces problèmes, en partant d'une annotation à plusieurs mains qui est progressivement unifiée afin d'obtenir un résultat objectif. Nous montrons que cette étape fait pleinement partie de l'étude linguistique elle-même.

This paper deals with problems related to discourse analysis within the framework of corpus linguistics, through a linguistic study dealing with procedurality in discourse. The fact that the study does not concern a specific lexical item makes it difficult to collect data without any predefined idea, in other words without introducing a bias in the study. The paper proposes a method to solve these problems, involving several annotators on the same texts and merging their proposals in order to get an objective unified annotation. We show that this step is a real part of the overall linguistic analysis.

\section{INDEX}

Mots-clés : analyse du discours, accord entre annotateur, annotation manuelle, procéduralité, recueil de données

Keywords : discourse analysis, data collection, inter-annotator agreement, manual annotation, procedurality

\section{AUTEURS}

\section{MARIE-PAULE JACQUES}

Université de Strasbourg / Laboratoire LILPA EA 1339

\section{THIERRY POIBEAU}

Laboratoire LaTTiCE, CNRS / ENS 